SCIENCE CHINA

Physics, Mechanics \& Astronomy

\title{
Gravitational wave astronomy: chance and challenge to fundamental physics and astrophysics
}

\author{
ZhouJian Cao* \\ Gravitational Wave and Cosmology Laboratory, Department of Astronomy, Beijing Normal University, Beijing 100875, China
}

Received August 17, 2016; accepted August 19, 2016; published online September 21, 2016

Citation: $\quad$ J. Cao, Gravitational wave astronomy: chance and challenge to fundamental physics and astrophysics, Sci. China-Phys. Mech. Astron. 59, 110431
(2016), doi: 10.1007/s11433-016-0324-y

Expected for a long time, the direct detection of gravitational wave (GW) has been realized by LIGO. Till now, two GW events, GW150914 [1] and GW151226 [2], have been detected. The success of LIGO is based on both the tremendous development of experiment technology [3-6] and theoretical research improvement in the past decades [7-9].

Regarding to GW150914, we are some lucky. The signal is so strong that advanced data analysis technology is not necessary to catch the signal (Figure 1), although the advanced data analysis can improve the signal to noise ratio (SNR) and confidence level strongly $[7,8]$. Regarding to GW151226, the signal is much weaker than that of GW150914. Without the advanced data analysis technology, the signal is completely invisible (Figure 1). In contrast, the advanced data analysis technology digs out the signal from the strong noise with SNR 13 and confidence level 5 $\sigma$ (Figure 2) [2]. GW151226 is a good example showing that the detection of GW is the result of combination of experiment achievement and theoretical research progress, if the propagation effect of $\mathrm{GW}$ on detector response can be ignored [10].

In order to make the advanced data analysis method work, gravitational wave template is needed. And the template strongly depends on the specific theoretical model of GW source. For example both GW150914 and GW151226 depend on the effective one body numerical relativity (EOBNR) model strongly $[7,8]$. Compact object binary coalescence $(\mathrm{CBC})$ is the major and most important transient sources for

*Corresponding author (email: zjcao@amt.ac.cn)
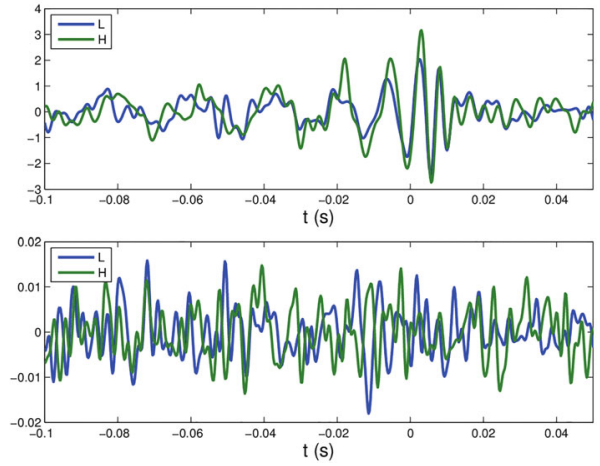

Figure 1 (Color online) Detected data of LIGO after whiten and band pass treatment. The top row and the bottom row correspond to GW150914 and GW151226 respectively. L and H mean the Livingston detector and the Hanford detector respectively.

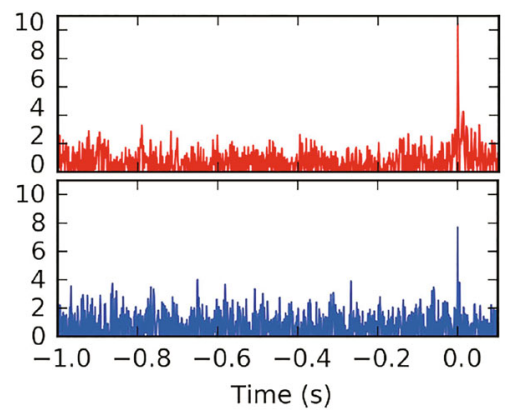

Figure 2 (Color online) Matched SNR for GW151226 (From Abbott et al. [2]). The top row and the bottom row correspond to the Hanford detector and the Livingston detector respectively. The matched SNR for Hanford and Livingston respectively is about 11 and 8, which leads to the total SNR $\sqrt{11^{2}+8^{2}} \approx 13[9]$. 
GW within the frequency range between $0.1 \mathrm{nHz}$ and $1 \mathrm{kHz}$ which could be detected by pulsar timing array, space-based or ground-based detectors. But constructing theoretical models of such GW sources is a highly non-trivial task $[7,8]$. In order to construct the specific theoretical model for GW sources, we need to solve the corresponding Einstein equations. Respect to strong GW radiation, the space-time region involved is highly dynamical and admits extremely strong gravitational field. Numerical method is necessary to treat such problems. But even numerical calculation itself is highly non-trivial. Numerical relativists used more than 40 years to make numerical calculation of Einstein equations stable [8]. Thanks to the breakthrough [11] and the following developments [8] of numerical relativity, many achievements about the GW source modeling have been got in the past years. Although the achievements about the theory of numerical calculation of Einstein equations, GW source models such as EOBNR, extremely large kick velocity of binary black hole and others, $\mathrm{CBC}$ with eccentricity and general spin configuration are far from well modeled. While the sources with eccentricity and/or general spin configurations are important sources for space-based and ground-based detectors.

The research about gravity is well known that experiment is lacking especially for strong and dynamical gravity region. Now, the GW detection brings us a hope to use GW signal as an experimental probe to study fundamental physics. But the difficulty is the information involved in fundamental theory is much more than that apparently carried by GW. So how to decode such an inverse problem is the challenge GW astronomy provides to us. Currently, the idea is firstly solving the direct problem to construct the corresponding theoretical model for GW sources based on specific fundamental physics law. Then based on such theoretical model, the matured GW data analysis technique, matched filtering method, can be adapted to treat the corresponding inverse problem [12]. The invoked difficulty is how to construct corresponding theoretical model for GW data analysis end. Leaving alone the numerical stability problem, the more serious problem is how to relate the matter field involved in the fundamental physical law to the astrophysical objects [12]. On the experiment side, there is also a challenge about how to determine the polarization modes [13]. But on the contrary, this is also a chance for fundamental physics, because it is possible to use GW detection to reveal the mystery of fundamental physics.

GW150914 means the direct detection of gravitational wave is possible. GW151226 implies that the number of GW sources for ground-based detectors is large enough for astronomy research. But in any case, including pulsar timing array and space-based detectors, the GW astronomy will be realized sooner or later. One point here also deserves to be pointed out that we only considered SNR larger than 13 now (GW151226 admits 13 and GW150914 admits 24).
Based on the primary design, if the SNR is larger than 8, the detection is trustable. So we can believe that more and more GW events will be detected by LIGO afterwards. Correspondingly, we can expect that the GW astronomy even with ground-based detectors alone will also be rich. The GW astronomy will open a new window for astronomer to observe our Universe. But it will be more wonderful if we can combine the traditional electromagnetic means and GW observation to realize multi-messenger astronomy. Such combination will reveal the mysteries such as gamma ray burst, fast radio burst and others [14]. But the time duration of GW event is short in general and the position location with GW itself is quite bad. These features make it difficult letting electromagnetic observations follow the GW detection promptly. This presents a great challenge to realize GW multi-messenger astronomy [15].

In summary, the exciting GW astronomy era has come. But in order to make the new GW astronomy grow fruitfully, we are faced with a lot of challenges which are related to fundamental physics and astrophysics. We believe such chances and challenges will make fundamental physics and astrophysics develop much faster in the near future.

1 B. Abbott, et al. (LIGO Sci Collaboration and Virgo Collaboration), Phys. Rev. Lett. 116, 061102 (2016).

2 B. Abbott, et al. (LIGO Sci Collaboration and Virgo Collaboration), Phys. Rev. Lett. 116, 241103 (2016).

3 D. Blair, L. Ju, and Z. H. Zhu, Sci. China-Phys. Mech. Astron. 58, 120401 (2015).

4 D. Blair, L. Ju, C. N. Zhao, L. Q. Wen, Q. Chu, Q. Fang, R. G. Cai, J. R. Gao, X. C. Lin, D. Liu, L. A. Wu, Z. H. Zhu, D. H. Reitze, K. Arai, F. Zhang, R. Flaminio, X. J. Zhu, G. Hobbs, R. N. Manchester, R. M. Shannon, C. Baccigalupi, W. Gao, P. Xu, X. Bian, Z. J. Cao, Z. J. Chang, P. Dong, X. F. Gong, S. L. Huang, P. Ju, Z. R. Luo, L. E. Qiang, W. L. Tang, X. Y. Wan, Y. Wang, S. N. Xu, Y. L. Zang, H. P. Zhang, Y. K. Lau, and W. T. Ni, Sci. China-Phys. Mech. Astron. 58, 120402 (2015).

5 V. P. Mitrofanov, S. Chao, H. W. Pan, L. C. Kuo, G. Cole, J. Degallaix, and B. Willke, Sci. China-Phys. Mech. Astron. 58, 120404 (2015), arXiv: 1602.05021.

6 D. Blair, L. Ju, C. N. Zhao, L. Q. Wen, H. X. Miao, R. G. Cai, J. R. Gao, X. C. Lin, D. Liu, L. A. Wu, Z. H. Zhu, G. Hammond, H. J. Paik, V. Fafone, A. Rocchi, C. Blair, Y. Q. Ma, J. Y. Qin, and M. Page, Sci. China-Phys. Mech. Astron. 58, 120405 (2015), arXiv: 1602.05087.

7 R. G. Cai, Z. J. Cao, and W. B. Han, Sci. Bull. 61, 1525 (2016).

8 Z. J. Cao, Z. H. Du, C. Y. Lin, and H. J. Yo, Sci. Sin.-Phys. Mech. Astron. in press (2016).

9 H. M. Lee, E. O. Le Bigot, Z. H. Du, Z. X. Lin, X. Y. Guo, L. Q. Wen, K. S. Phukon, V. Pandey, S. Bose, X. L. Fan, and M. Hendry, Sci. China-Phys. Mech. Astron. 58, 120403 (2015), arXiv: 1602.05573.

10 Z. Chang, C. G. Huang, and Z. C. Zhao, Sci. China-Phys. Mech. Astron. 59, 100421 (2016).

11 F. Pretorius, Phys. Rev. Lett. 95, 121101 (2005).

12 Z. Cao, P. Galaviz, and L. F. Li, Phys. Rev. D 87, 104029 (2013).

13 C. G. Huang, Y. G. Li, and N. Zhu, Chin. Phys. Lett. 33, 080402 (2016).

14 H. Gao, X. Ding, X. F. Wu, Z. G. Dai, and B. Zhang, ApJ 807, 163 (2015), arXiv: 1506.06816.

15 X. L. Fan, Sci. China-Phys. Mech. Astron. 59, 640001 (2016). 